\title{
Soft curvature sensors for measuring the rotational angles of mechanical fingers
}

(C) The Author(s) 2020. This article is published with open access at link.springer.com and journal.hep.com.cn

\begin{abstract}
The design, fabrication, and testing of soft sensors that measure elastomer curvature and mechanical finger bending are described in this study. The base of the soft sensors is polydimethylsiloxane (PDMS), which is a translucent elastomer. The main body of the soft sensors consists of three layers of silicone rubber plate, and the sensing element is a microchannel filled with galliumindium-tin (Ga-In-Sn) alloy, which is embedded in the elastomer. First, the working principle of soft sensors is investigated, and their structure is designed. Second, the relationship between curvature and resistance is determined. Third, several sensors with different specifications are built in accordance with the structural design. Experiments show that the sensors exhibit high accuracy when the curvature changes within a certain range. Lastly, the soft sensors are applied to the measurement of mechanical finger bending. Experiments show that soft curvature sensors can effectively reflect mechanical finger bending and can be used to measure the bending of mechanical fingers with high sensitivity within a certain working range.
\end{abstract}

Keywords soft sensor, Ga-In-Sn alloy, strain sensing, curvature sensing, mechanical finger bending

\section{Introduction}

Flexible electronic devices have developed rapidly in recent years [1-3]. As an important type of flexible electronic devices, soft sensors, such as soft tactile [4-6] and soft curvature sensors, have been developed consistently by researchers. Soft sensors have many advantages over rigid ones. Soft sensors have high adaptability, long

Received January 16, 2020; accepted June 8, 2020

Haixiao LIU, Li LI ( $₫)$, Zhikang OUYANG, Wei SUN

School of Mechatronic Engineering and Automation, Shanghai University, Shanghai 200444, China

E-mail: lili.shu@shu.edu.cn service life, and low cost. Moreover, they have many structural forms and can be arranged flexibly in accordance with the site environment [7]. As a type of soft sensor, soft curvature sensors can be used to detect the bending of human joints, such as hands, elbows, shoulders, and even small muscle vibration. These sensors sense human action information and transform the obtained information into electrical signals in accordance with certain rules, thus providing original data for subsequent information processing and information fusion analysis. Currently, soft curvature sensors are used as the core devices of electronic skin [8-10], wearable electronic equipment [11-13], and flexible human-computer interaction equipment [14].

The performance of typical soft curvature sensors is determined by three factors, namely, conductors, substrates, and distributions of functional structures. The flexible sensing unit converts angles into physical signals that can be directly measured, such as voltage, resistance, and capacitance. Converting angles to resistance signals is a popular option.

Resistive materials exert a critical impact on the performance of soft curvature sensors. These sensors have strict requirements on conductor flexibility. Compared with solid metal materials [15], liquid conductors have no fixed shape, and their shape is related to the shape of the container; thus, they meet the needs of soft curvature sensors. Common liquid conductors include liquid metals and ionic solutions, and many researchers have explored their application to soft sensors.

Liquid metal is one of the conductor materials used in soft sensors. Dickey et al. [16] studied the rheological behavior of liquid metal eutectic gallium-indium (eGaIn) and injected this material into microfluidic channels to form stable microstructures of liquid metal. Kramer et al. [17] developed a fabrication method that utilizes masked deposition and selective wetting to produce hyperelastic electronic circuits. The electronic circuits are composed of a thin elastomer film embedded with microchannels of liquid-phase GaIn alloy. Dickey [18] designed a stretchable and soft electronic sensor by using liquid metals, thus 
promoting the application of liquid metals in soft sensors.

Another type of conductor material used in flexible sensors is ionic liquid. Zhang et al. [19] developed a highly stretchable and transparent strain sensor based on ionic liquids. The high conductivity of 1-ethyl-3-methylimidazolium bis ionic liquids was used $[19,20]$. Xu et al. [21] designed a new silicon-based strain and force sensor with biocompatible conductive liquids. The conductive liquids were potassium iodide (KI) and glycerol solution (Gly). Choi et al. [22] proposed a highly stretchable, low-cost strain sensor using an extremely cost-effective ionic liquid of ethylene glycol/sodium chloride. Measuring the resistance of ionic solutions requires intermediate frequency alternating current (AC), which might limit the application of this sensor.

The resistance range of ionic liquids changes from $1 \times$ $10^{-4}$ to $1 \Omega$, which exerts a favorable effect on the accuracy of soft sensors. However, performing measurements by introducing AC, especially in the actual application of soft sensors, is difficult. On the contrary, measuring the resistance of liquid metals commonly involves direct current (DC), which is beneficial for the application of portable soft sensors. In this study, a liquid metal composed of Ga-In-Sn is used as a conductor in a soft curvature sensor. The melting point of liquid metal Ga$\mathrm{In}-\mathrm{Sn}$ is lower than that of eGaIn. Thus, a liquid environment can be easily reached at room temperature, and this fluidity is suitable for the application of soft curvature sensors. A detailed comparison of different conductor materials is presented in Table 1.

The substrate is another important factor that affects the performance of soft sensors, and silicone rubber is one of the commonly used materials. Majidi et al. [23] studied the effect of different elastomers on the performance of sensors. Kramer et al. [17] investigated the method of combining liquid metals with elastomers. Given the mechanical properties and adaptability of silicone elastomer, this material is used as the substrate to design a soft curvature sensor in the current study.

The structural design of soft sensors is also an important research issue that affects sensor performance [24]. Case et al. [25] conducted mechanical measurements of three representative elastomers used in soft robotic systems. They found that the microchannel determines the shape of the sensing unit and is the basis of realizing different functions of a soft sensor. Yuen and Kramer [26] fabricated microchannels on elastomeric substrates. Majidi et al. [23] designed a soft curvature sensor by using stress concentration; this sensor changes the cross-sectional area of the microchannel without changing the length during operation. White et al. [27] designed several sensors with different functions and precision by changing the length and arrangement of sensor microchannels. By superimposing two microchannels, White et al. [28] built a device that can identify positive curvature, negative curvature, and strain. The structure of these sensors is precise and well-executed, but the structural complexity causes installation difficulties. For example, the width of several mechanical fingers is insufficient, and the application of these sensors is difficult. In the current study, three layers of silicone rubber sheet are used with serpentine microchannels. This structure can guarantee high sensor sensitivity and is suitable for the rotational measurement of large curvature.

The purpose of this study is to develop a soft curvature sensor that can be used to measure the rotational angles of mechanical fingers effectively. The soft sensor has the advantages of simple structure, high sensitivity, easy fabrication, and flexible deployment.

The remainder of this article is organized as follows. The bending theory of soft curvature sensors is established in Section 2. The structural design of soft curvature sensors is presented in Section 3. The production of the proposed soft curvature sensor is introduced in Section 4, and the properties of the sensor are investigated in Section 5. Section 6 presents experiments conducted on the soft curvature sensor to measure the bending of mechanical fingers. The conclusions are provided in Section 7.

\section{Bending theory of soft curvature sensors}

During measurement, the bending of soft sensors can be viewed as a combination of sensor tensile compression and shear deformation. When a body bends, its internal parts change. With an isotropic homogeneous elastomer as an example, the layers near the outer side undergo tensile deformation when the elastomer has pure bending deformation, and the layers near the inner side undergo compressive deformation. The layer in the middle is called

Table 1 Comparison of different conductor materials

\begin{tabular}{|c|c|c|c|c|}
\hline Material type & Material & Resistivity/( $\Omega \cdot \mathrm{m})$ & Detection mode & Reference \\
\hline Solid metal & Constantan foils & $4.80 \times 10^{-7}$ (typical) & DC & [15] \\
\hline \multirow[t]{2}{*}{ Liquid metal } & Liquid-phase Ga-In alloy & $2.94 \times 10^{-7}$ (typical) & DC & {$[16,17]$} \\
\hline & Ga-In-Sn & $2.89 \times 10^{-7}$ (typical) & DC & Method proposed in this study \\
\hline \multirow[t]{2}{*}{ Ionic liquid } & 1-ethyl-3-methylimidazolium bis & $2.40 \times 10^{-4}$ (typical) & $\mathrm{AC}$ & {$[19,20]$} \\
\hline & KI-Gly & 7.40 (typical) & $\mathrm{AC}$ & [21] \\
\hline
\end{tabular}


the neutral layer, and it is neither stretched nor compressed.

When an elastomer bends, each part is stretched or compressed to a certain extent. Ignoring the thickness change in the elastomer while bending, the curvature $\kappa$ of each layer can be obtained using the strain $\varepsilon$ of the elastomer and distance $h$ between the layers and the neutral layer. Their relationship can be expressed as

$$
\varepsilon=h \kappa .
$$

Assume that a conductor exists in the elastomer, and its volume is constant. The shape of the conductor changes with the shape of the elastomer. A schematic of elastomer bending is displayed in Fig. 1. The resistance of the conductor varies with its shape. If the resistance is known, then the curvature and strain of the elastomer in which the conductor resides can be obtained.

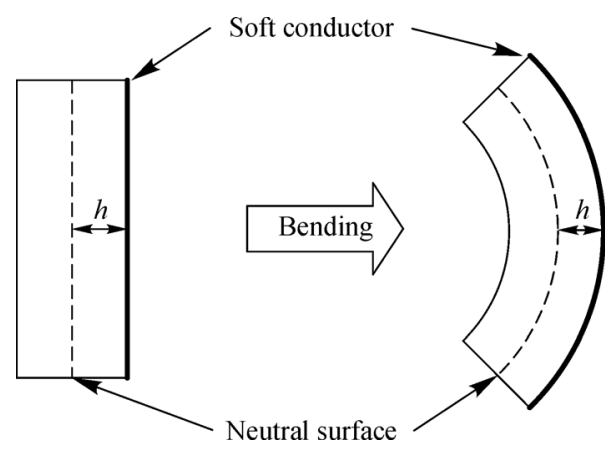

Fig. 1 Schematic of elastomer bending.

Given that the length of the conductor varies in direct proportion to the length of the elastomer in which the conductor is located, the length of the conductor can be determined in accordance with the length of layer in which the conductor is located.

Suppose that volume $V$ of the conductor embedded in the elastomer is constant, and its cross-sectional area is $A$ with an initial value of $A_{0}$, and the length is $L$ with an initial value of $L_{0}$. Then,

$$
V=L A=L_{0} A_{0} .
$$

According to the law of resistance, the resistance $R$ of a conductor is proportional to its length $L$ and inversely proportional to its cross-sectional area $A$. This relation can be expressed as

$$
R=\rho \frac{L}{A},
$$

where $\rho$ is the resistivity of the conductor. The crosssectional area $A$ of the conductor after bending is

$$
A=\frac{V}{L}=\frac{L_{0} A_{0}}{L}=\frac{L_{0} A_{0}}{L_{0}+\Delta L},
$$

where $\Delta L$ is the length change of the conductor. According to the definition of strain, $\Delta L=\varepsilon L_{0}$. Meanwhile, incorporating Eq. (1) into Eq. (4) results in the new equation:

$$
A=\frac{A_{0}}{1+h \kappa} .
$$

By substituting Eq. (5) into the resistance equation of Eq. (3), resistance can be expressed as

$$
R=\rho \frac{L_{0}}{A_{0}}(1+h \kappa)^{2} .
$$

Initial resistance $R_{0}$ can be obtained with

$$
R_{0}=\rho \frac{L_{0}}{A_{0}} .
$$

The variation in resistance is $\Delta R=R-R_{0}$, and the relationship between resistance change and strain can be described by

$$
\Delta R=\rho \frac{L_{0}}{A_{0}}\left(2 h \kappa+h^{2} \kappa^{2}\right) .
$$

Therefore, Eq. (8) shows the theoretical relationship between curvature and resistance.

\section{Structural design of a soft curvature sensor}

The structure of a soft sensor determines its performance. Soft curvature sensors have good flexibility; thus, their substrates should also have good flexibility. Silicone rubber is a new type of polymer material with high elasticity, and it is used as the base of the proposed soft sensor. The combustion product of silicone rubber is silicon dioxide, which is an insulator. A microchannel is embedded inside the sensor. The conductor must be filled with microchannels to convert sensor change into resistance change. Ga-In-Sn alloy, a type of metal that is liquid at room temperature $\left(20^{\circ} \mathrm{C}\right)$, is selected as the filler for the microchannels. The main components of Ga-In-Sn alloy are $68.5 \% \mathrm{Ga}, 21.5 \% \mathrm{In}$, and $10 \% \mathrm{Sn}$. Its melting point is from $6^{\circ} \mathrm{C}$ to $12{ }^{\circ} \mathrm{C}$, and the electrical conductivity is $3.46 \times 10^{6} \mathrm{~S} / \mathrm{m}$ at $20^{\circ} \mathrm{C}$.

Equation (8) shows that when the soft curvature sensor is bent, the greater the distance is between the conductor and neutral surface of the measured object, the greater the change in resistance is. An appropriate distance must be ensured to obtain sufficient resistance variation. However, the thickness of the soft sensor is another factor to be considered. An increase in the thickness of the soft sensor means a corresponding increase in the distance between the conductor and neutral surface of the body to be measured. Flexibility is affected when the thickness of the soft sensor exceeds a certain limit. Therefore, the distance of the conductor from the neutral surface must be limited to within a certain range. 
The parameters of microchannels filled with liquid alloys determine the resistance of soft sensors. A large microchannel length or a small cross-sectional area equates to a high resistance of the soft sensor and a large amount of resistance change when the same bending occurs. Therefore, increasing the length of the sensor microchannel or reducing its cross-sectional area is beneficial for increasing the resolution of soft sensors. Soft sensors have a limited size, but the length of microchannels can be effectively increased by using serpentine channels in a limited area. Moreover, the dense arrangement of microchannels increases production difficulty. Therefore, the factors mentioned above need to be considered comprehensively to achieve good performance of soft sensors.

Soft sensors may also encounter extreme situations. When a soft sensor is over-bent, the pressure of the liquid metal inside the sensor increases sharply, causing damage to the soft sensor. Therefore, certain measures must be implemented to reduce risks. Both ends of the proposed soft sensor are non-sensing areas that are used to fasten the sensor, and two cavities are set on them. Compared with the thickness of the other parts, the thickness of the silicone rubber above them is increased by once. When the liquid pressure in the microchannel increases, the liquid in the microchannel flows into the cavities to ensure that the sensor does not undergo structural damage under extreme conditions. The cavities on both sides of the soft sensor are responsible for connecting the internal microchannels to the external wires. Therefore, a large cavity volume could increase the contact surface of the external wires with liquid metal, reduce the contact resistance, and improve the measurement accuracy. As shown in Fig. 2, the sensor consists of substrate cavities, a microchannel, and external wires. The substrate is silicone rubber, and the sensing element is Ga-In-Sn alloy.

\section{Production of the soft curvature sensor}

As shown in Fig. 3, several sensors are fabricated in accordance with the determined structure. Their microchannel lengths and cross-sectional areas vary. Each end of the microchannel has a cavity. Silicone rubber sheets manufactured according to national standards are selected to maximize the sensor accuracy under limited conditions.

Two sizes of silicone rubber plates, namely, thicknesses of 1 and $0.5 \mathrm{~mm}$, are selected. The microchannel is filled with Ga-In-Sn alloy with $99.99 \%$ purity. The fabrication process is divided into the following sub-processes: Cutting the silicone rubber, bonding the silicone rubber sheets, injecting the liquid metal, extracting the wire interface, and sealing the soft sensor.

The fabrication of soft curvature sensors can be divided into six steps, as shown in Fig. 4. In Step 1, the silicone rubber sheet is cut and cleaned simply before production to prevent dust from affecting the seal of the soft sensor. The silicone rubber plate is cut into substrates with $140 \mathrm{~mm}$ length and $25 \mathrm{~mm}$ width. Each thick silicone rubber sheet is matched with two thin silicone rubber sheets. The thick silicone rubber sheet is used as the bottom layer, and the two thin silicone rubber sheets are utilized as the middle and upper layers.
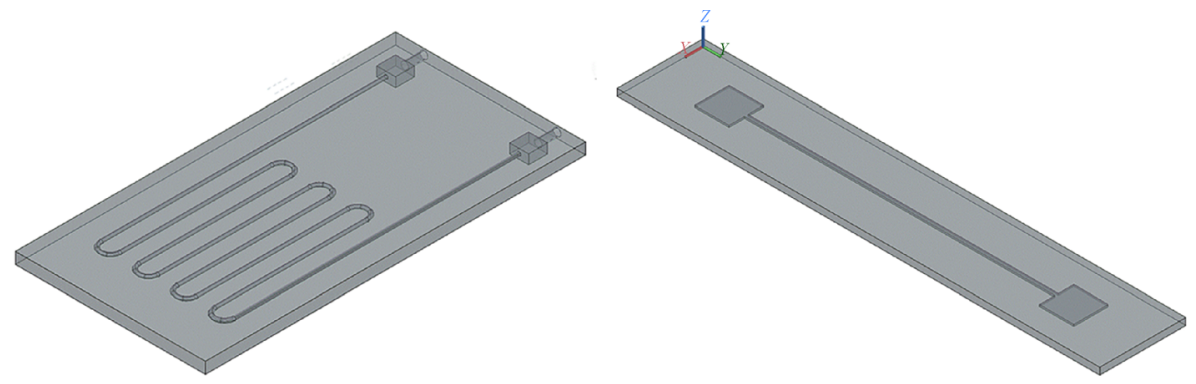

Fig. 2 Comparison of the structural design of serpentine and linear microchannels.

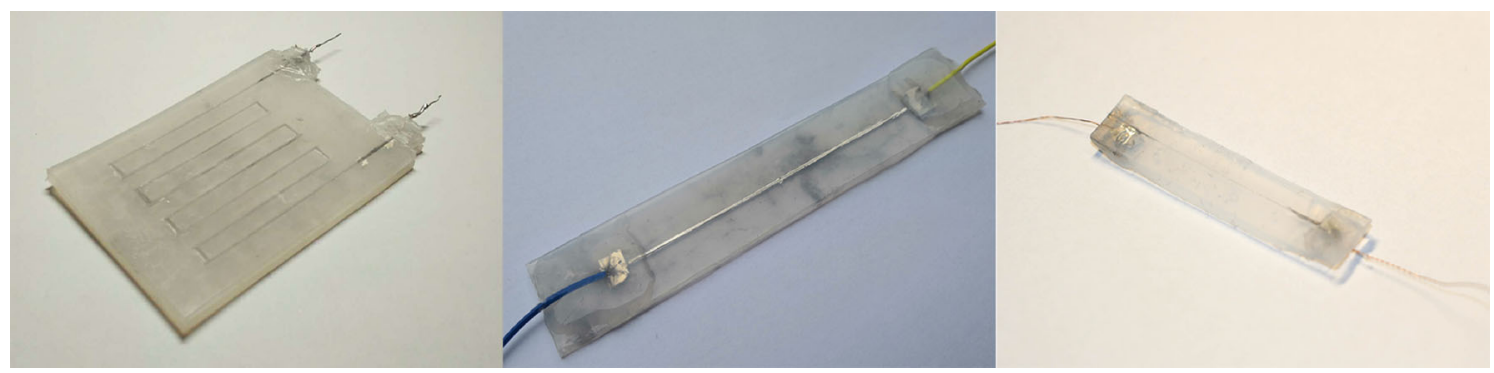

Fig. 3 Aerial view of the soft curvature sensors. 


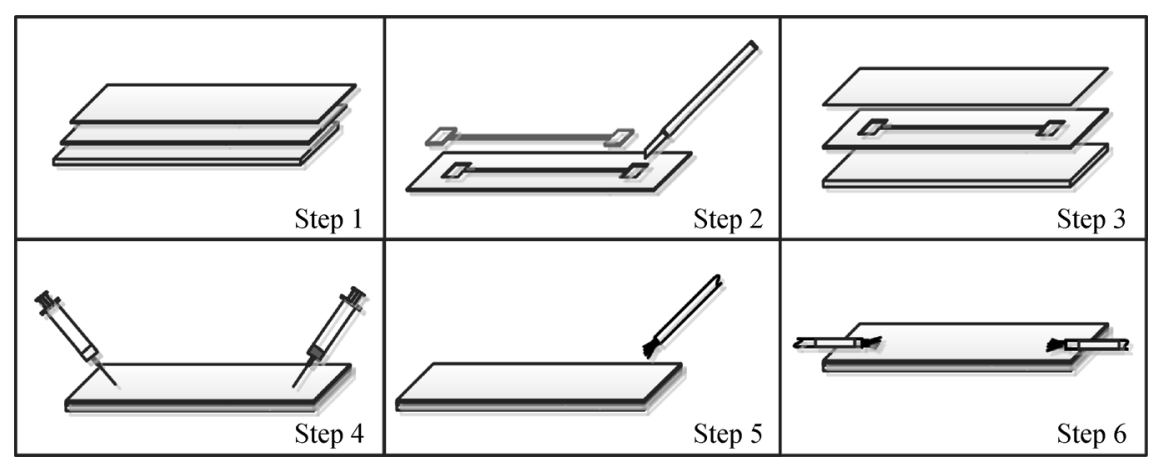

Fig. 4 Production of a soft curvature sensor.

The microchannel is designed in Step 2. The microchannel is located in the middle layer of the soft sensor. The middle layer is cut in the manner shown in Fig. 4, and the middle part is discarded. The depth of the microchannel is not an issue because the thickness of the silicone rubber sheet is certain. What needs to be considered is the width of the microchannel.

In Step 3, three layers of silicone rubber sheets are glued together. Notably, the silicone rubber sheet is soft. If an improper bonding method is used or the force applied is not uniform, then the evenness of the final microchannel width will be affected. In the process of pasting, the width of the microchannel should be adjusted to meet the design requirements as much as possible. Under the condition of ensuring that the position of the middle layer does not change, the sensor is placed at room temperature for 24 hours to complete the curing of the adhesive. After curing, the top silicone rubber sheet is firmly adhered to the middle layer. The glue between the upper and middle layers should be used appropriately. Excessive use of the glue will cause it to spill into the microchannel during the pasting process, thus blocking the microchannel. On the contrary, if the glue used is insufficient, a gap could develop between the upper and middle layers. Under the action of external forces, liquid alloys may overflow from the microchannels and leak out. Therefore, the amount of glue used must be appropriate. Twenty-four hours is allowed to pass until the adhesive is fully cured while ensuring that the paste is complete and that the microchannel is not blocked.

In Step 4, the Ga-In-Sn alloy is injected. The air in the microchannel is pumped out using a syringe to completely fill the microchannel with liquid alloy. Then, the liquid alloy is injected to the microchannel. The atmospheric pressure forces the liquid alloy to fill the entire microchannel because of the pressure difference inside and outside the microchannel. In addition, bubbles are avoided.

In Step 5, the external wires are connected. Connecting external conductors to internal liquid conductors is difficult. A flexible wire is used as the external conductor to increase the contact area between the external conductor and internal liquid conductor. When creating the micro- channel, a cavity is reserved on both sides of the microchannel, which is filled with liquid alloy, and the external conductors are inserted as far into the cavity as possible to ensure full contact between the liquid alloy and external metal wires.

In Step 6, the electrical interface is sealed, and the sealing object is the joint part between the sensor body and the wire. The silicone rubber adhesives evenly cover the cavities and wires, and the gluing surface becomes smooth under the action of gravity. Glue solidification marks the completion of the entire process of making the soft curvature sensor. No bubbles should appear in the glue; otherwise, the sealing quality would be affected.

The selection of glue for the production of a soft curvature sensor is extremely important. Silicone rubbers cannot be bonded with ordinary glue because of its excellent chemical inertia, and special silicone rubber adhesives must be used. Usually, two transparent silicone rubber adhesives are selected: Acetoxysilicon and alkoxysilicon. Alkoxysilicon adhesive is likely to produce bubbles in the solidification process, and these bubbles seriously affect the sealing of the soft sensor. Therefore, acetoxysilicon adhesive is selected in the current study to bond the soft sensor.

\section{Properties of the soft curvature sensor}

\subsection{Method of measuring curvatures}

As shown in Eq. (1), a certain relation exists between curvature and strain, which can transform into each other under certain conditions. When the curvature of the neutral surface of the measured object is known, the value of $h$ (the distance from the conductor to the neutral plane) determines the curvature. The test of measuring curvature is converted into a test of measuring strain to reduce the complexity of experimentation. The sensor is stretched unilaterally, and its resistance change is measured. The device used for measuring resistance is the HELPASS HPS2513S DC micro-resistance tester.

A change in the sensor's cavity considerably affects the 
sensor output. Two pieces of cloth soaked with liquid silicone rubber are pasted on both ends of the soft sensor in order to improve the stability of the sensor; this distributes the force to the main body of the soft sensor and stabilizes the interface between the external wires and liquid conductor. The main body of the soft sensor is partially removed to achieve improved performance. For experiment convenience, one end of the sensor is fixed, and oneway tension is applied to the other end.

The contact resistance between the external wires and liquid metal is inevitable. Therefore, the influence of contact resistance should be considered in the experiment. This study assumes that contact resistance does not change during the stretching and releasing of the soft sensor, and the constant $c$ represents the portion of the microchannel's conductive liquid resistance versus total resistance. Equation (8) can be rewritten as

$$
\Delta R=c R_{\text {total }}\left(2 h \kappa+h^{2} \kappa^{2}\right),
$$

where $\kappa$ is the curvature of the measured object, and $R_{\text {total }}$ is the sum of sensor resistance $R_{0}$, external wire resistance $R_{\mathrm{w}}$, and contact resistance $R_{\mathrm{c}}$. Substituting Eq. (1) into Eq. (9) yields

$$
\Delta R=c R_{\text {total }}\left(2 \varepsilon+\varepsilon^{2}\right)
$$

5.2 Experiments on the characteristics of soft curvature sensors

The strain response is measured by stretching the sensor to a known length. A pointer is made from the wires on the active end of the soft sensor body, as displayed in Fig. 5. A coordinate paper is placed on a desktop with grids that are $1 \mathrm{~mm}$ apart. The pointer is matched with the printed line on the coordinate paper to obtain a given extension. Extensions of 10, 20, 30, and $40 \mathrm{~mm}$ are tested. The results show that the sensor still works effectively even when the strain is greater than $45 \%(40 \mathrm{~mm})$. For equipment safety, the strain during testing is limited to less than $45 \%$. The experiment is limited to static loading conditions because several studies have shown that the response of an elastomer sensor based on liquid metal is usually separated from the loading rate [5].

The $10 \mathrm{~mm}$ tensile test is performed on the soft sensor with a length of $135 \mathrm{~mm}$, width of $23 \mathrm{~mm}$, and height of $2 \mathrm{~mm}$ to investigate the measurement characteristics of the soft sensor. According to the parameters of the microchannel, the theoretical resistance of this soft curvature sensing unit is $0.092 \Omega$. The initial resistance of the sensor, $R_{\text {total }}$, is measured 20 times, and the average resistance is $0.247 \Omega$. The measured value is obviously larger than the theoretical one due to many reasons. One of the influencing

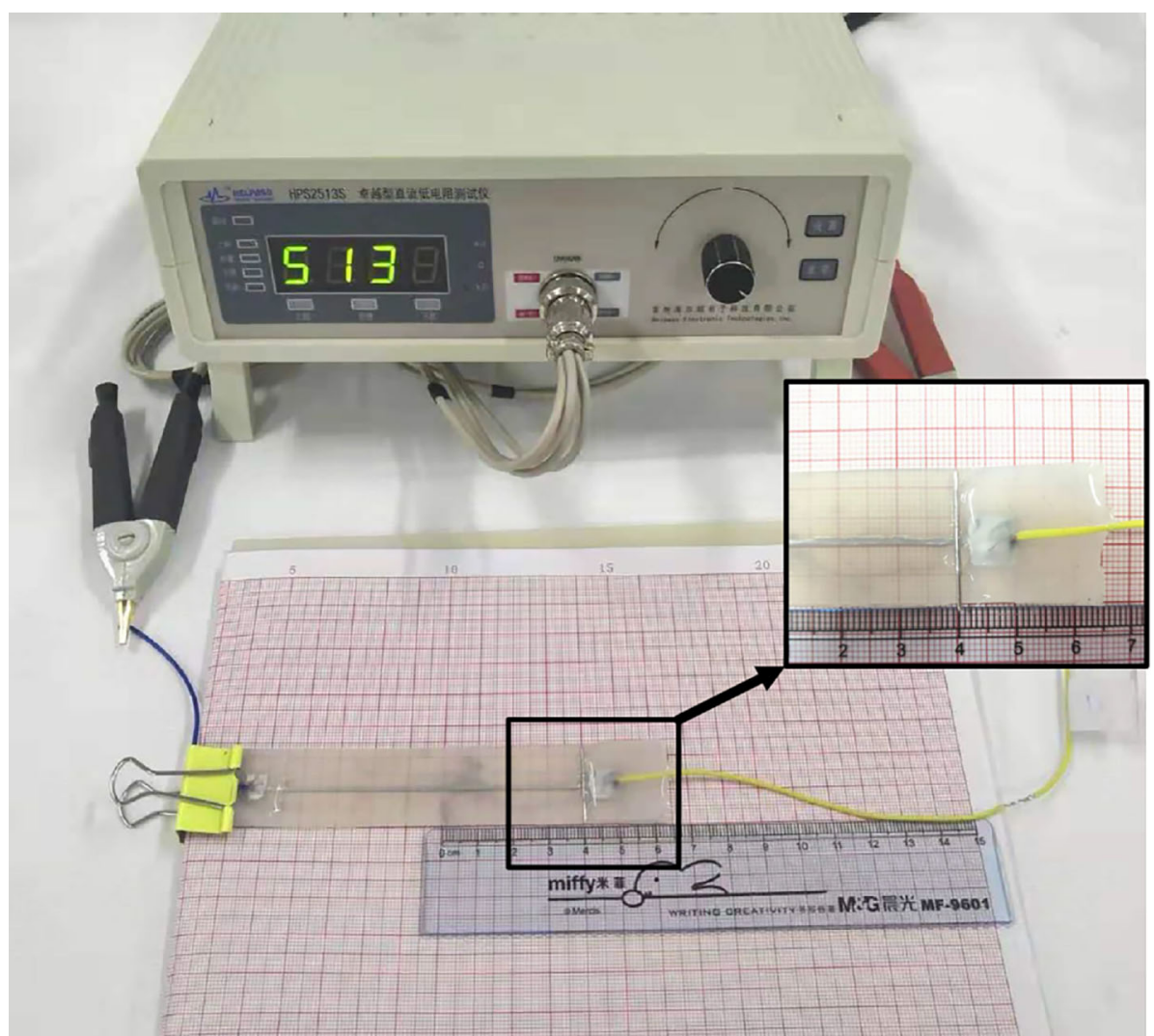

Fig. 5 Experimental platform for the stretching of a soft curvature sensor. 
factors is the manufacturing accuracy of sensor microchannels. A glue solidification point may exist in the microchannels, resulting in a reduced cross-sectional area. In addition, the measurement resistance includes wire, contact, and sensing unit resistance. The maximum strain of the soft curvature sensor is $11.2 \%$ (stretched by $10 \mathrm{~mm}$ ). The theoretical and measured values of its resistance change are shown in Fig. 6. The maximum relative deviation between the measured value and theoretical fitting line is $6.9 \%$, and its linearity is $\pm 6.88 \%$. The measured value is always higher than the theoretical one, and as the strain increases, the relative deviation is almost unchanged. Compared with Eq. (10), this is considered the fact that the actual resistance of $c R_{\text {total }}$ is greater than the theoretical resistance. The curve of the measured value shows that the slope of the curve increases suddenly then returns to normal as the strain of the soft sensor increases. This phenomenon stems from the error accumulation caused by the accuracy of measuring equipment.

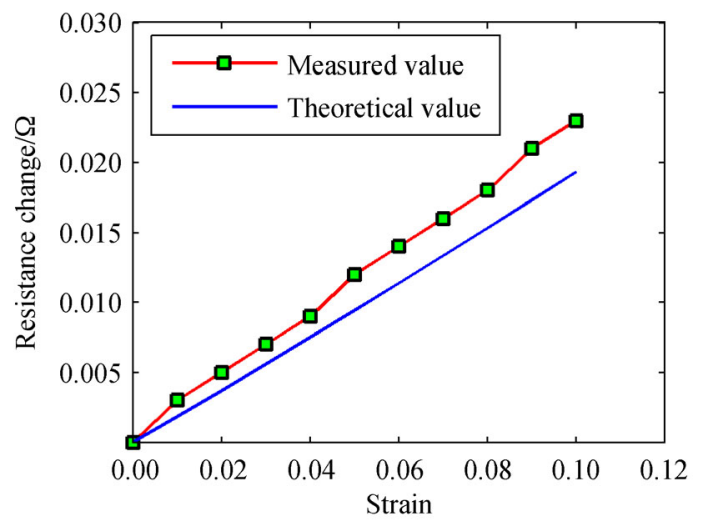

Fig. 6 Resistance change in the soft curvature sensor when stretched by $10 \mathrm{~mm}$ (strain: 11.2\%).

A sensor is usually reused many times. Tensile experiments using 10,20 , and $30 \mathrm{~mm}$ are performed 20

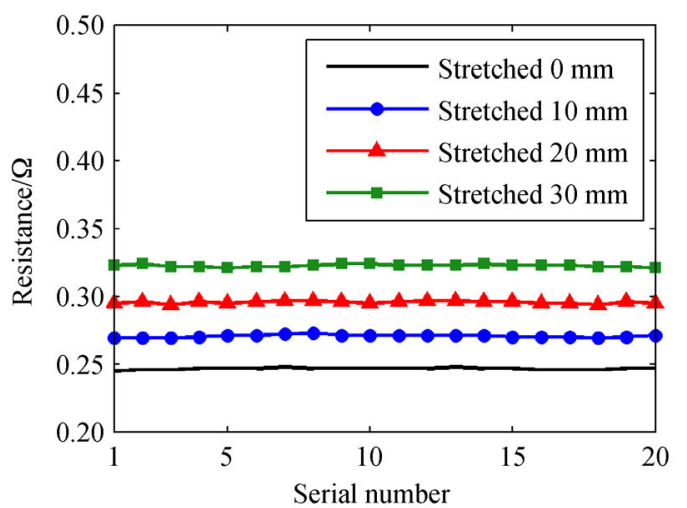

(a) times each to investigate the effect of repeated use on the soft sensor. In between measurements, the strain of the sensor is restored to zero for benchmarking. The experiment results are shown in Fig. 7. When the strain is zero, the mean value of the measured resistance is $0.2467 \Omega$, and the standard deviation is $0.0007 \Omega$. The standard deviations of data for 10,20 , and $30 \mathrm{~mm}$ stretching are $11 \times 10^{-4}, 9 \times 10^{-4}$, and $9 \times 10^{-4} \Omega$, respectively. In Fig. 7(a), the solid line presents the initial resistance of the unstretched sensor, and the denoted line presents the resistance of the stretched sensor.

The data are generally distributed in gentle lines, but fluctuations are observed in certain segments. The trends of several fluctuations on these two lines show consistencies. The deviation is caused by the fluctuation in the measuring instrument. Resistance variation is important in studying the characteristics of sensors. The data are drawn on a graph, and the results are shown in Fig. 7(b). Visually, the two lines stretched short are smoother than that long one. The standard deviations of the three lines are $7.1 \times 10^{-4}$, $6.9 \times 10^{-4}$, and $12 \times 10^{-4} \Omega$. Compared with the data on $30 \mathrm{~mm}$ stretching, the data on the short stretching lengths are more concentrated. One of the reasons is that the resistance of the soft sensor is relatively low, and the movement of the external wires influences the measurement results. Moreover, the stress produced by the sensor is large because of the large stretching length; thus, jitter could be produced during measurement. In consideration of the instrument, the data of $\Delta R$ are highly representative of the actual performance of the soft sensor.

Normally, soft curvature sensors perform measurements for a long time. Therefore, the hysteresis of soft sensors must be investigated. The loading-unloading experiment is an important method to test the performance of equipment. In this study, a sensor initial resistance of $0.247 \Omega$ is used as the reference to stretch the sensor by $30 \mathrm{~mm}$ multiple times, and the change in resistance during the stretching process is recorded. The result is shown in Fig. 8. The hysteresis error of the soft curvature sensor is

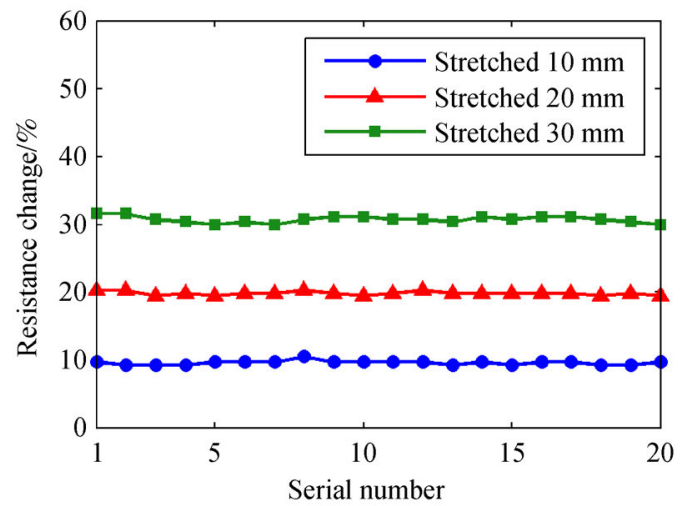

(b)

Fig. 7 Resistance performance under different stretching lengths for repeated experiments: (a) Stretched by 10, 20, and $30 \mathrm{~mm}$; (b) comparison of the stretched sensor. 


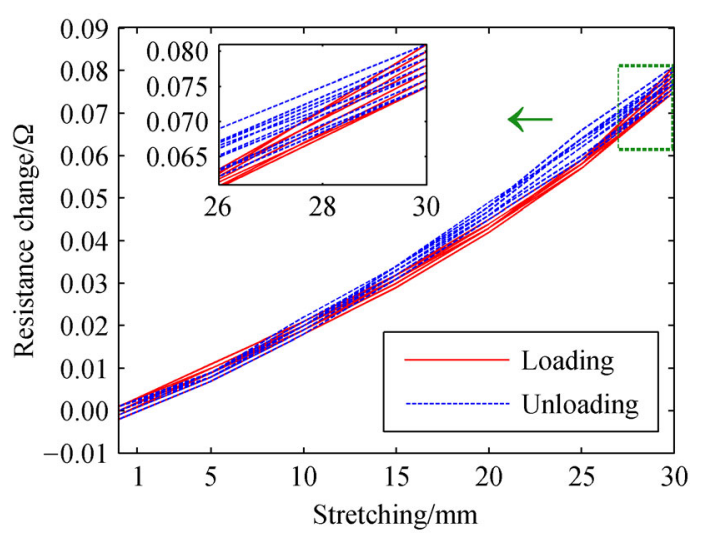

Fig. 8 Resistance performance of the soft curvature sensor under loading and unloading conditions.

small and within a small stretching range $(0-10 \mathrm{~mm})$. The hysteresis error increases with the increase in tensile length. In the range of $20-30 \mathrm{~mm}$, the hysteresis error increases rapidly, and the maximum hysteresis error is $3.9 \%$. The maximum deviation between the output values of each measuring point in forward and reverse strokes is $0.0070 \Omega$. The average resistance under $30 \mathrm{~mm}$ stretching is taken as the fullness value, and the repeatability error is $\pm 9.21 \%$. Therefore, the strain of the soft curvature sensor should be limited to a certain range during sensor application if high accuracy is required. Otherwise, the error can be increased appropriately.

In summary, the soft curvature sensor performs well, and it exhibits high measurement accuracy when the stretch is not more than $10 \%$. This result indicates that the curve of $\Delta R$ versus $\Delta L$ can be established according to the geometric structure of the sensor microchannel and the resistivity of the conductor. The sensor can be used for special conditions. For example, when a mechanical finger is bent, the change in the length of the fingertip is related to the rotational angle of the joint, and the change is small. Therefore, this soft curvature sensor is suitable for the measurement of finger bending.

\section{Experiments on the soft curvature sensor for measuring the bending of mechanical fingers}

Given the sensor's working principle, the sensor can be used to measure not only the curvature of elastomers but also the rotational angle of rigid structures, such as fingers.

The part above the metacarpal knuckle of a single finger is investigated in this study. In accordance with the anatomy of the hand, the structures of the index, middle, ring, and little fingers are similar. Each finger consists of three knuckles and three joints. Each multi-phalange finger can perform movement in a bending finger manner with only one tendon actuator. The motions between joints are correlated. Therefore, each finger can be measured by one sensor.

For measurement convenience and repeatability, a manipulator, instead of a human hand, is used to perform finger bending experiments. The experiment platform and the placement of the soft curvature sensor are shown in Fig. 9(a). The soft curvature sensor is fixed on the mechanical finger, and the rotation of the finger joint is controlled by the operating interface of a computer. Its output is measured and displayed by a micro-resistance tester. The revolute pairs are used as joints of the manipulator fingers. In the case of finger bending, the length of the finger bent backward increases. As shown in Fig. 10, the relationship between the joint rotational angle and length variation $\Delta L$ of the mechanical finger bent backward can be expressed as

$$
\Delta L=\omega r,
$$

where $r$ represents the radius of the revolute pair and $\omega$ denotes the radian at which the joint rotates. Combining Eqs. (11) and (10) results in

$$
\Delta R=c R_{\text {total }}\left[2 \omega \frac{r}{L_{0}}+\left(\omega \frac{r}{L_{0}}\right)^{2}\right] .
$$

Equation (12) demonstrates the relationship between rotational radian $\omega$ of the revolute joint and resistance change $\Delta L$. It also indicates that the angle of joint can be obtained easily if $\Delta L$ is known.

The four-bar linkage is utilized in the mechanical finger driving device. If the motion of one finger joint is obtained, then the motion of another joint can be calculated using the relationship of the four-bar linkage mechanism.

The sensor can be arranged flexibly in accordance with the site environment. The length and width of the mechanical finger limits the size of the soft sensor. Hence, a sensor with a similar structure but reduced size is placed on the mechanical finger. As shown in Figs. 9(b) and 9(c), the distal finger joint is selected as the object to be measured. The two ends of the soft curvature sensor are fixed on two adjacent phalanges by Velcro.

To measure the rotational angle of mechanical finger, the joint is rotated, and the corresponding change of resistance is recorded. The resistance of the rotational angle at $0^{\circ}$ is considered the initial resistance. With every $2^{\circ}$ as a unit of rotation, the joint rotates one unit per time. The relationship between rotational angle and resistance change is calculated with Eq. (12). The experimental results are shown in Fig. 11.

Figure 11 indicates that the measured values (the line denoted by squares) are lower than the theoretical ones (the solid line). Many factors can lead to this situation. The main possible reason is that the parameters of the microchannel deviate from the designed values in the manufacturing process. Within $30^{\circ}$ to $70^{\circ}$, the trend of the measured values is consistent with that of the theoretical 


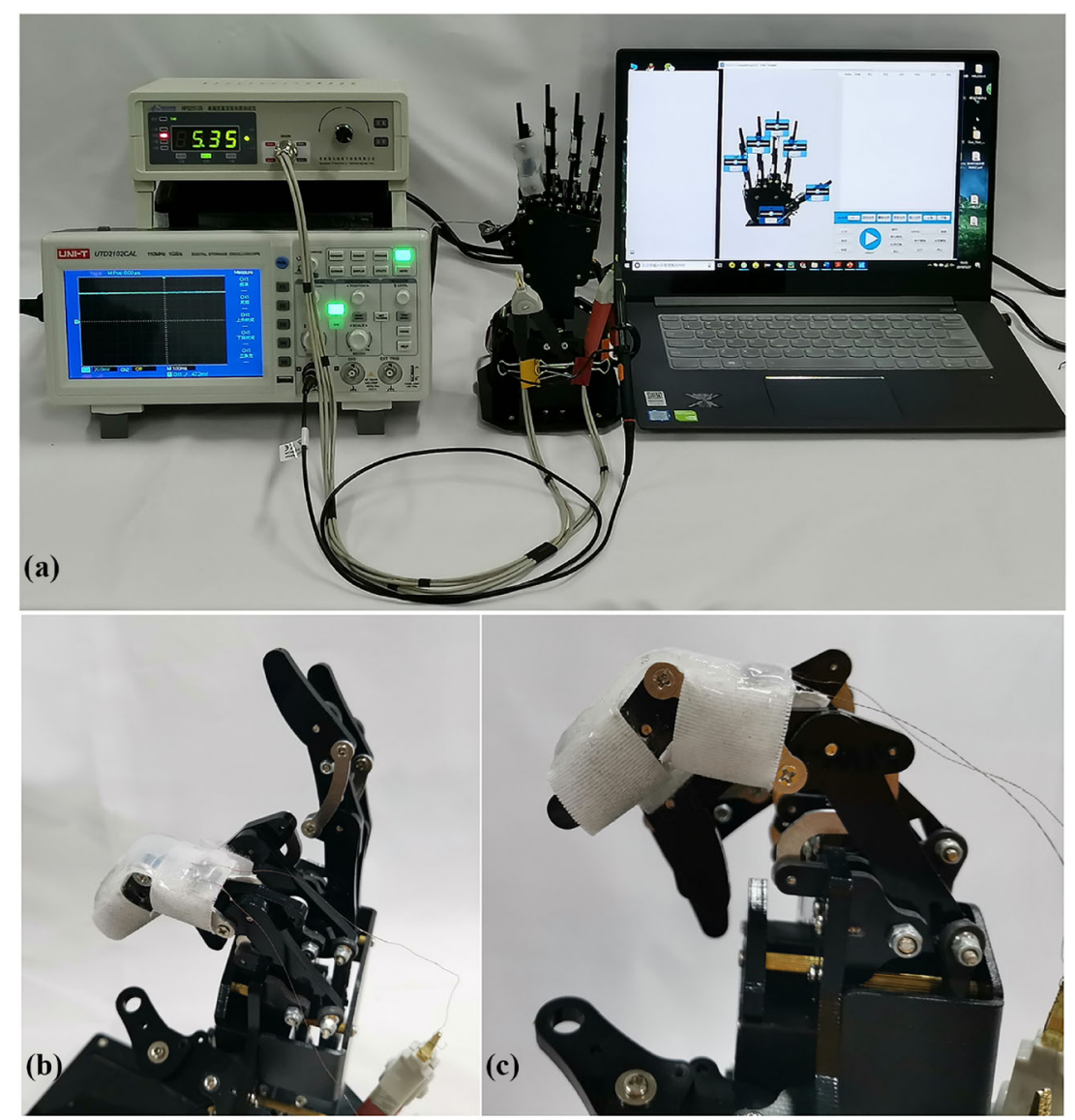

Fig. 9 Experimental platform for measuring mechanical fingers with the soft curvature sensor: (a) Experimental platform, (b) large bending angle, and (c) small bending angle.

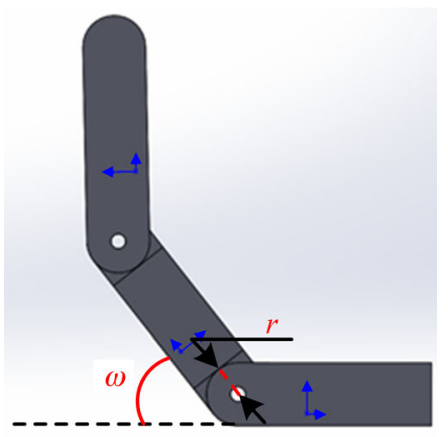

Fig. 10 Diagram of a mechanical index finger.

values, signifying that the soft curvature sensor has high accuracy. However, when the rotational angle is small, the accuracy of the measured values of the soft curvature sensor decreases due to measurement difficulty, leading to considerable deviation between the measured and theoretical values. Compared with the soft curvature sensor used in the tensile experiment, the soft sensor used in this experiment has a smaller size and less deformation, resulting in a larger measurement deviation in this

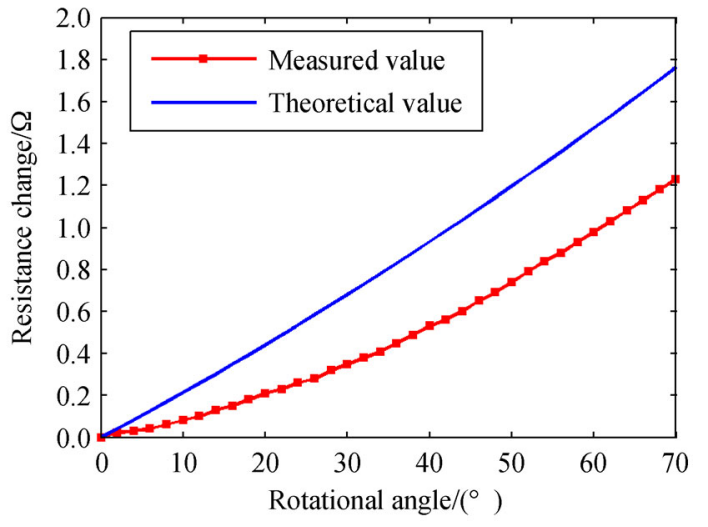

Fig. 11 Performance of the soft curvature sensor when the mechanical finger rotates.

experiment than that in the tensile experiment.

A regression analysis is also performed to quantify the response of the soft sensor. The response equation of the soft sensor is assumed to be a quadratic function, and the software MATLAB is used for calculation. The results are achieved as Eq. (13): 


$$
\begin{aligned}
\Delta R= & -3.5 \times 10^{-3}+7.27 \times 10^{-3} \theta \\
& +1.4 \times 10^{-4} \theta^{2},
\end{aligned}
$$

where $\theta$ is the rotational angle with a unit of ${ }^{\circ}$. This rotational angle is shown in Fig. 10 as $\omega$ with a unit of rad, and the unit of resistance is $\Omega$.

The measured and fitted values are compared, as shown in Fig. 12. The scatter points represent the experimental observations, and the dotted lines represent $95 \%$ confidence intervals (CI). Figures 12(a)-12(c) show local graphs of different intervals for better observation. The fitting line achieves good results, and all points are within 95\% CI. The deviation between the theoretical and experimental $\Delta R$ is shown in the histogram in Fig. 13. The deviation has a normal distribution, indicating that the fitting effect is effective.

The experiment is repeated, and a set of data is obtained to verify the accuracy of the fitting curve. Equation (13) is used to determine the angle of each measurement, and the results are shown in Fig. 14. The solid line represents the theoretical values, and the dotted line represents the experimental observations.

As plotted in Fig. 14, the deviation between the

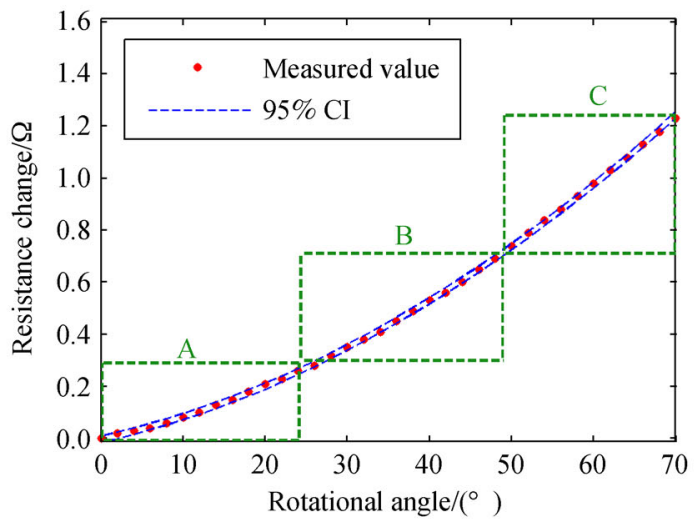

(a)

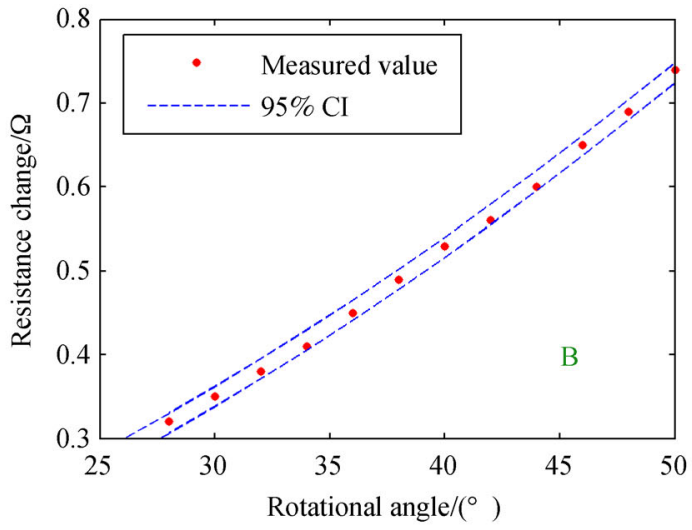

(c) experimental observation and theoretical value is not obvious at a small rotational angle. The deviation increases when the rotational angle increases. The maximum deviation is $2.9 \%$ at $80^{\circ}$. In general, the experimental observations fluctuate slightly around the actual value.

\section{Conclusions}

A soft curvature sensor is proposed, and its characteristics are investigated. Experiments show that the maximum relative deviation between the measured value of strain and the theoretical fitting line is $6.9 \%$, and the hysteresis error of the soft curvature sensor is small when the strain of the sensor is less than $11.2 \%(10 \mathrm{~mm})$. When the strain is increased from $11.2 \%$ to $33.6 \%$, the precision decreases, and the maximum hysteresis error increases to $3.9 \%$. Moreover, the proposed soft sensor is applied to the measurement of mechanical finger bending. The deviation between the experimental and theoretical values is not obvious at a small rotational angle. The maximum deviation is $2.9 \%$ at $80^{\circ}$. Therefore, the design of the soft curvature sensor is feasible, and this type of soft sensor

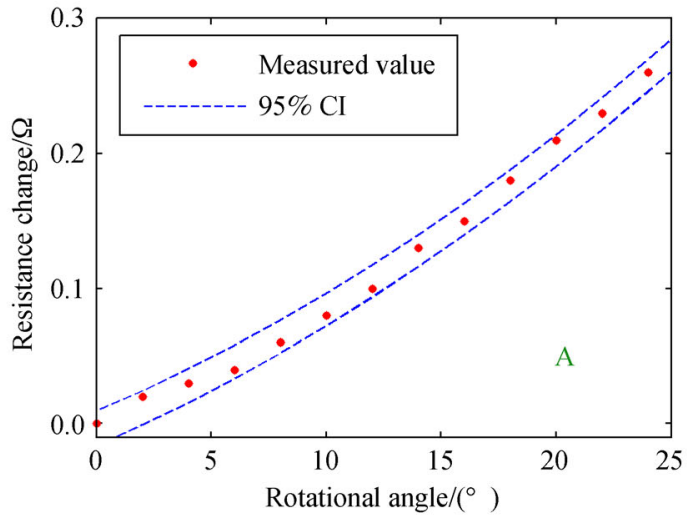

(b)

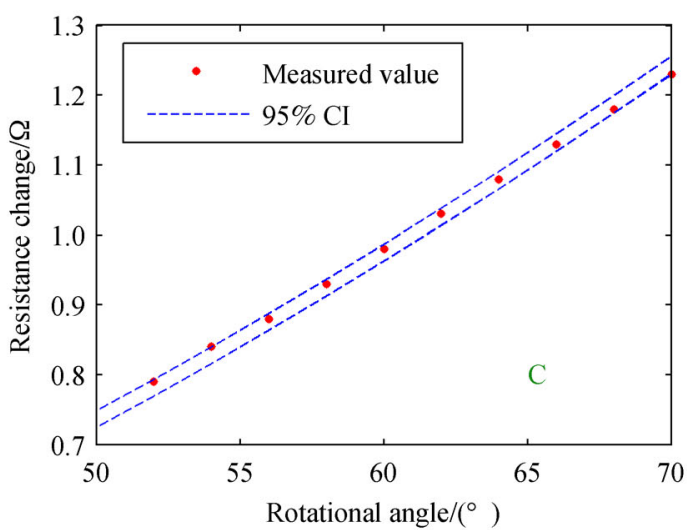

(d)

Fig. 12 Distribution of resistance changes of the soft curvature sensor versus the rotational angle of the mechanical finger: (a) Global curve; curve segments of (b) A, (c) B, and (d) C noted in (a). 


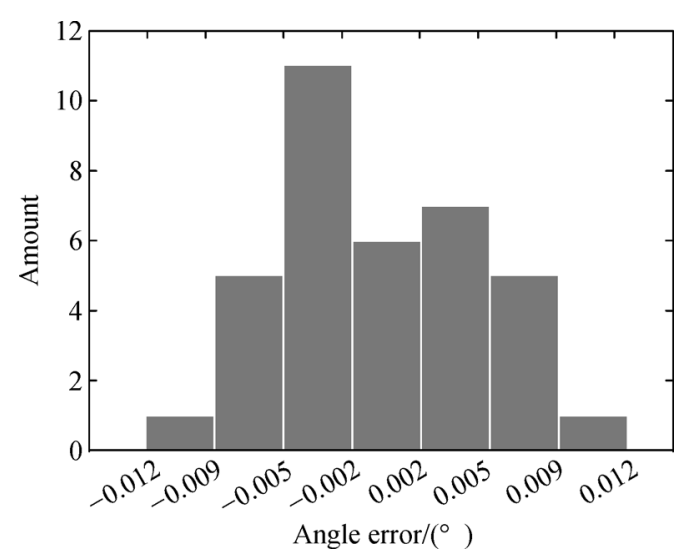

Fig. 13 Histogram of the deviation between theoretical and experimental $\Delta R$.

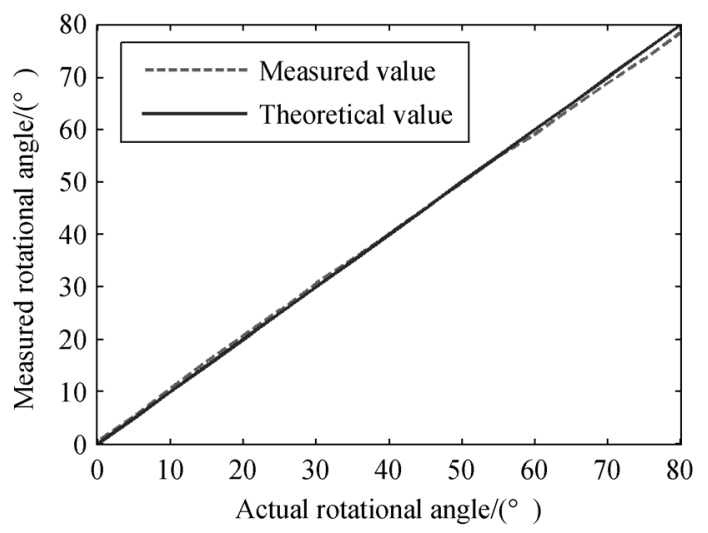

Fig. 14 Comparison of the measured rotational angle and theoretical values for repeated experiments.

can be used to measure the rotational angles of mechanical fingers effectively.

Acknowledgements This work was supported by the National Natural Science Foundation of China (Grant No. 51405280).

Open Access This article is licensed under a Creative Commons Attribution 4.0 International License, which permits use, sharing, adaptation, distribution and reproduction in any medium or format, as long as you give appropriate credit to the original author(s) and the source, provide a link to the Creative Commons licence, and indicate if changes were made.

The images or other third party material in this article are included in the article's Creative Commons licence, unless indicated otherwise in a credit line to the material. If material is not included in the article's Creative Commons licence and your intended use is not permitted by statutory regulation or exceeds the permitted use, you will need to obtain permission directly from the copyright holder.

To view a copy of this licence, visit http://creativecommons.org/licenses/ by $/ 4.0 \%$.

\section{References}

1. Lopes P A, Paisana H, De Almeida A T, et al. Hydroprinted electronics: Ultrathin stretchable $\mathrm{Ag}-\mathrm{In}-\mathrm{Ga}$ E-skin for bioelectronics and human-machine interaction. ACS Applied Materials \& Interfaces, 2018, 10(45): 38760-38768

2. Cai J D, Xia Q, Luo Y J, et al. A variable-width harmonic probe for multifrequency atomic force microscopy. Applied Physics Letters, 2015, 106(7): 071901

3. Hellebrekers T, Kroemer O, Majidi C. Soft magnetic skin for continuous deformation sensing. Advanced Intelligent Systems, 2019, 1(4): 1900025

4. Kramer R K, Majidi C, Wood R J. Wearable tactile keypad with stretchable artificial skin. In: Proceedings of 2011 IEEE International Conference on Robotics and Automation. Shanghai: IEEE, 2011, 1103-1107

5. Park Y L, Chen B R, Wood R J. Design and fabrication of soft artificial skin using embedded microchannels and liquid conductors. IEEE Sensors Journal, 2012, 12(8): 2711-2718

6. Hammond F L, Kramer R K, Wan Q, et al. Soft tactile sensor arrays for force feedback in micromanipulation. IEEE Sensors Journal, 2014, 14(5): 1443-1452

7. Xu S, Zhang Y H, Jia L, et al. Soft microfluidic assemblies of sensors, circuits, and radios for the skin. Science, 2014, 344(6179): 70-74

8. Hammock M L, Chortos A, Tee C K, et al. 25th Anniversary Article: The evolution of electronic skin (E-skin): A brief history, design considerations, and recent progress. Advanced Materials, 2013, 25(42): 5997-6038

9. Qiu L, Cao J G, Zhou J H, et al. Research progress of flexible and elastic bionic E-skin for robot. Journal of Central South University (Science and Technology), 2019, 50(5): 1065-1074 (in Chinese)

10. Cao J G, Zhou J H, Miao C X, et al. Research progress and development strategy on tactile sensors for E-skin. Journal of Harbin Institute of Technology, 2017, 49(1): 1-13 (in Chinese)

11. Patel S, Park H, Bonato P, et al. A review of wearable sensors and systems with application in rehabilitation. Journal of Neuroengineering and Rehabilitation, 2012, 9(1): 21

12. Mardonova M, Choi Y. Review of wearable device technology and its applications to the mining industry. Energies, 2018, 11(3): 547

13. Amjadi M, Kyung K U, Park I, et al. Stretchable, skin-mountable, and wearable strain sensors and their potential applications: A review. Advanced Functional Materials, 2016, 26(11): 1678-1698

14. Wan A B, Heo S, Ji S, et al. Transparent and flexible fingerprint sensor array with multiplexed detection of tactile pressure and skin temperature. Nature Communications, 2018, 9(1): 2458

15. Liu H, Zhao H Y, Li S, et al. Adhesion-free thin-film-like curvature sensors integrated on flexible and wearable electronics for monitoring bending of joints and various body gestures. Advanced Materials Technologies, 2019, 4(2): AR1800327

16. Dickey M D, Chiechi R C, Larsen R J, et al. Eutectic galliumindium (eGaIn): A liquid metal alloy for the formation of stable structures in microchannels at room temperature. Advanced Functional Materials, 2008, 18(7): 1097-1104

17. Kramer R K, Majidi C, Wood R J. Masked deposition of galliumindium alloys for liquid-embedded elastomer conductors. Advanced Functional Materials, 2013, 23(42): 5292-5296

18. Dickey M D. Stretchable and soft electronics using liquid metals. 
Advanced Materials, 2017, 29(27): 1606425

19. Zhang S H, Li J J, Wang F X, et al. Wearable wide-range strain sensors based on ionic liquids and monitoring of human activities. Sensors, 2017, 17(11): 2621

20. Chossat J B, Tao Y, Duchaine V, et al. Wearable soft artificial skin for hand motion detection with embedded microfluidic strain sensing. In: Proceedings of IEEE International Conference on Robotics and Automation. Seattle: IEEE, 2015, 2568-2573

21. Xu S, Hsu W H, Vogt D M. Biocompatible soft fluidic strain and force sensors for wearable devices. Advanced Functional Materials, 2019, 29(7): 1807058

22. Choi D Y, Kim M H, Oh Y S, et al. Highly stretchable, hysteresisfree ionic liquid-based strain sensor for precise human motion monitoring. ACS Applied Materials \& Interfaces, 2017, 9(2): 1770 1780

23. Majidi C, Kramer R K, Wood R J. A non-differential elastomer curvature sensor for softer-than-skin electronics. Smart Materials \&
Structures, 2011, 20(10): 105017

24. Xia Q, Shi T L, Liu S Y, et al. Shape and topology optimization for tailoring stress in a local region to enhance performance of piezoresistive sensors. Computers \& Structures, 2013, 114-115: 98-105

25. Case J C, White E L, Kramer R K. Soft material characterization for robotic applications. Soft Robotics, 2015, 2(2): 80-87

26. Yuen M C, Kramer R K. Fabricating microchannels in elastomer substrates for stretchable electronics. In: Proceedings of ASME International Manufacturing Science \& Engineering Conference. New York: ASME, 2016, V002T01A014

27. White E L, Case J C, Kramer R K. Multi-element strain gauge modules for soft sensory skins. IEEE Sensors Journal, 2016, 16(8): 2607-2616

28. White E L, Case J C, Kramer R K. Multi-mode strain and curvature sensors for soft robotic applications. Sensors and Actuators A: Physical, 2017, 253: 188-197 